\title{
Exploiting of Classification Paradigms for Early diagnosis of Alzheimer's disease
}

\author{
G Stalin Babu ${ }^{1}$, S.N.Tirumala Rao², R Rajeswara $\mathrm{Rao}^{3}$ \\ ${ }^{1}$ Assistant Professor, Dept of CSE, Aditya institute of technology and management, Tekkali \\ ${ }^{1}$ Research scholar, Dept of CSE, JNTUK, Kakinada \\ ${ }^{2}$ Professor, Dept of CSE, Narasaraopeta Engineering College, Narasaraopeta. \\ ${ }^{3}$ Professor, Dept of CSE, JNTUK University College of Engineering, Vizianagaram
}

\begin{abstract}
Alzheimer's disorder is an incurable neurodegenerative disease that ordinarily affects the aged population. Coherent automated assessment methods are essential for Alzheimer's disease diagnosis in early from distinct images modalities using Machine Learning. This article focuses on exploring various feature extraction and classification methods for early detection of AD proposed by researchers and proposes a modern predictive model that includes Voxel based Texture analysis of brain images for extract features and Optimized Classifier Deep Convolution Neural Network (DCNN) employed for enhance accuracy.
\end{abstract}

Keywords: Alzheimer's disease (AD), DCNN, Feature Extraction.

\section{Introduction}

Alzheimer's disease (AD), a type of dementia is portrayed by revolutionary thinking problems and behavior, beginning in the middle or old age. Alzheimer's disease leads to poor memory, perception, and Behavior. In year 1907 Alois Alzheimer's German neuropathologist first described about Alzheimer's [1]. The pathological characteristics of neuritis are brain plaques and explicit brain degeneration cells. In general, the symptoms develop slowly and get serious enough to be interfere in everyday life. While the Oldness is a paramount risk factor, AD is not merely a sickness of old age. In time, Alzheimer's disease gets worse and is fatal, Alzheimer's disease is difficult to recognizing by Doctors before reaches to clinical stage by reason of diagnosis process done manually it leads to highly significance to error due to interference of perception of patients, even if warning signs are not recognized by patients themselves [2].

Dementia is the general brain disorder the most common, progressive and debilitating brain disease of which is Alzheimer's disease. It breaks down brain cells, interfering significantly enough with memory, thinking, and behavior to influence the job, hobbies, and social life of a person. An assessment and analysis of the existing studies, number of common trends and shortcomings identified. The patterns most evident include a short one $\mathrm{AD}$ detection and forecast growth using the methods of machine learning. Machine learning also plays a vital role in diagnosis and prediction of AD. Among the bigger gaps was a too many attributes selection, use of Unconventional data set, class inequality, overtraining and inadequate objective assessments or Validation. However, the better designed and the better the Studies validated showed that machine learning approaches enhance AD accuracy.

Machine learning is expanding under Artificial intelligence, it's more powerful than standard statistical tools, better understanding of problem and consist of different tools for probabilistic, statistical decision depends on prior learning. Uses previous experience to identify current events and recognize existing patterns.

Alzheimer's disease is getting worse over time and fatal. In diagnosing of $\mathrm{AD}$ using Machine learning it consists of sequence of steps. The initial steps are preprocessing takes the unprocessed MRI contains of numerous artifacts, including intensity similarity, extra corpuscular tissue and more, step is important because it should be taken to make certain high accuracy in preceding steps. The second step in the diagnostic procedure is Feature extraction; a method that extracts peculiar features from pre-processed images in various anomalous groups therefore, "within class similarity is maximized and between class similarities is minimized". This image classification technique is capable of providing information on the existence anomalies of input brain image used to diagnose dementia and Alzheimer's., Brain image classification is an important process in diagnostic systems. The main aim of the classification process is to determine between distinct anomalousness of brain images predicated on the best possible feature set. [1].

Many traditional Machine Learning algorithms are available for classification, such as K-Nearest Neighbors (KNN), Naïve Bayes , Support Vector Machine (SVM), Random Forest (RF), Decision tree and more, which offers the best results for extraction of features used in the diagnosis of dementia and Alzheimer's disease.

In this article give a predictive model which includes novel feature extraction and hybrid optimal classifier DCNN for detection of Alzheimer's using MRI images.

\section{2. Alzheimer's Disease}


Alzheimer's Disease (AD) which is also known as Senile Dementia [3]. It's a progressive disease that causes brain cells to waste, degenerate and die., it is a uninterrupted decline in thinking, behavioral and mental skills that disrupts a person's capability to function on one's own. A person with this disease will develop severe memory impairment. There is no complete cure or alteration of this disease. Instead of curing, we can take several programs and treatments which maximize the better functionality of the brain.

\section{A. Symptoms:}

Loss of memory is that the main symptom of Alzheimer's disease. Recalling recent events or conversations is sometimes a tough early sign of the disease. Memory impairments worsen because the disease progresses, and different symptoms develop [4].

Changes in brain associated with Alzheimer's disease cause increasing problems with:

i. Loss of memory which disrupts everyday life

ii. Challenges in planning or solving problems

iii. Difficulty carrying out common roles

iv. Confusion of time and place

v. Misplacing things and lose the opportunity to retrace steps

vi. Mood shifts and attitude changes

vii. Wrong or poor judgment

\section{B. Stages:}

There are seven stages in the Alzheimer disease which helps to understand that the person is in which stage by its symptoms [5].

Stage 1: No Impairment: In this Stage No Symptoms, no memory problems and Alzheimer's disease are undetectable.

Stage 2: Very Mild Decline: during this stage dementia can be recognized by a clinical assessment or family members. They may forget where they left things, but person do well with memory.

Stage 3: Mild Cognitive Decline: At this stage, the family members, co-workers and friends of the aged may begin to identify difficulties. Duration of stage is seven years.

Stage 4: Moderate Cognitive Decline: In stage four of Alzheimer's, explicit symptoms of the disease are supposed. Maybe forgot about own personal history become moody

Stage 5: Moderately Severe Decline: starting of stage major memory gaps present, person need assistance with activities of day-by-day, unable to recollect phone number and address.

Stage 6: Severe Cognitive Decline: also called Middle Dementia, during this stage person need supervision symptoms are inability to identify closed friends and family members, experience loss of bowel and bladder control, and speech

Stage 7: Very severe cognitive decline: It is final stage in the Progress of AD, people will have lost their ability to speak or convey information. They regularly require assist with the majority of their exercises, which includes washing, toileting, dressing, eating, and different day- by- day exercises.

\section{Machine Learning:}

"Machine learning is subset of artificial intelligence it provides machine ability to learn automatically and improve from experience without explicitly programmer". It learns from previous experiences and build a Model when new data is comes easily predicts belongs to which category. Machine learning is statistical models, aim is to analyze and recognize structure of data [6]. The machine learning is help to analysis of data to diagnosis of Diseases in medical field. What to create good machine learning model it requires Data preprocessing capabilities, Algorithms, Automation and iterative process, scalability and Ensemble modeling and better Model gives high accuracy.

\subsection{Types of Machine Learning Techniques:} There are 4 types of machine learning Algorithms
A. Supervised learning
B. Unsupervised learning
C. Semi supervised Learning
D. Reinforcement Learning

\section{A. Supervised learning:}

Supervised learning is approach in which can be teach machine by labeled data. The outcome (dependent variable) is predicted from the previous experience (independent variables), it creates mapping function $\mathrm{Y}=\mathrm{f}(\mathrm{X})$ it maps input to expected output, where $\mathrm{X}$ input variable and $\mathrm{Y}$ is output variable [7]. It can help to get Optimized performance for predictions for new unlabelled data from experiences. Decision Tree, Regression, KNN, Random Forest are Supervised Learning approaches. Supervised learning has two types' a) classification b) Regression.

Classification is process of assign a class label to input data. There are two categories of classifications. One is Binary classifier involve two class labels either "YES" or "NO", another one is Multi label Classifier refers one or more class.

Regression is process of " Predict continuous outcome (dependent variable) from one or more input (independent variable), $\mathrm{Y}=\mathrm{bX}+\mathrm{c}$ where $\mathrm{X}$ is independent variable, $\mathrm{Y}$ is Dependent Variable and b, c is Co-efficient " .

\section{B. Unsupervised learning:}


Unsupervised learning is approaching the machine trained on unlabelled data without any supervisor, have not any outcome/target to estimate. it consists of input variable $\mathrm{X}$ and responding Output variable. K-means, Principal Component Analysis, Apriori algorithm for association rule learning are Unsupervised learning algorithms.

Unsupervised learning has categorized 2 types a) Clustering b) Dimensionality Reduction.

Clustering is process of group the objects based on homogenous characteristics. Clustering is based on Density, Centroid and Distribution.

Dimensionality Reduction method is minimizing the no of features there two components in one is feature selection and feature extraction.

\section{Semi supervised Learning:}

Semi supervised Learning is Combination of Supervised (less labeled data) and unsupervised (more Unlabelled data), in the process first cluster the data by using unsupervised learning then use labeled the data to label remain unlabelled data [8].

\section{Reinforcement Learning}

\begin{tabular}{|c|c|c|c|c|c|c|c|c|}
\hline \multirow{2}{*}{ Author } & \multirow{2}{*}{ Dataset } & \multirow{2}{*}{ Methodology } & \multirow{2}{*}{$\begin{array}{c}\text { Image } \\
\text { modality }\end{array}$} & \multirow{2}{*}{ Features } & \multirow{2}{*}{ Target } & \multicolumn{3}{|c|}{ Performance } \\
\hline & & & & & & Accuracy & Sensitivity & Specificity \\
\hline \multirow{2}{*}{$\begin{array}{c}\text { Peng et al. } \\
{[10]}\end{array}$} & \multirow{2}{*}{ ADNI } & \multirow{2}{*}{$\begin{array}{l}\text { SVM(multi } \\
\text { kernel) }\end{array}$} & \multirow{2}{*}{$\begin{array}{l}\text { sMRI,PET, } \\
\text { SNP }\end{array}$} & \multirow{2}{*}{$\begin{array}{c}\text { Volume + } \\
\text { Mean } \\
\text { intensity } \\
\text { features }\end{array}$} & $\mathrm{CN}$ vs $\mathrm{AD}$ & 96.1 & 97.3 & 94.9 \\
\hline & & & & & MCI vs.AD & 80.3 & 85.6 & 69.8 \\
\hline $\begin{array}{c}\text { Kar et al. } \\
{[11]}\end{array}$ & ADNI & ANN & sMRI,CT & ROI & $\mathrm{CN}$ vs.AD & 100 & 100 & 100 \\
\hline $\begin{array}{c}\text { Sheng et al. } \\
{[12]}\end{array}$ & ADNI & SVM & fMRI & RF-Score & $\mathrm{CN}$ vs.AD & 95.8 & & \\
\hline $\begin{array}{c}\text { Spasov et al. } \\
{[13]}\end{array}$ & ADNI & $\mathrm{CNN}$ & MRI & $\begin{array}{c}\text { ROI, } \\
\text { APOe4 }\end{array}$ & $\begin{array}{c}\text { sMCI } \\
\text { vs. } \\
\text { pMCI }\end{array}$ & 92.5 & 86.5 & 85 \\
\hline $\begin{array}{c}\text { Fritsch et al. } \\
{[14]}\end{array}$ & $\begin{array}{c}\text { Pitt } \\
\text { Corpus }\end{array}$ & LSTM & Linguistic & n-gram & $\mathrm{CN}$ vs.AD & 85.6 & & \\
\hline \multirow{3}{*}{$\begin{array}{c}\text { Gosztolya et al. } \\
{[15]}\end{array}$} & \multirow{3}{*}{ ADNI } & \multirow{3}{*}{ SVM -Linear } & \multirow{3}{*}{$\begin{array}{c}\text { Acoustic } \\
\text { signal }\end{array}$} & \multirow{3}{*}{ MFCC } & CN vs MCI & 80 & & 75.9 \\
\hline & & & & & $\mathrm{CN}$ vs $\mathrm{mAD}$ & 86 & & 87.5 \\
\hline & & & & & $\begin{array}{l}\text { MCI vs } \\
\text { mAD }\end{array}$ & 80 & & 85.7 \\
\hline $\begin{array}{c}\text { Krishnakumar } \\
\text { Vaithinathan et } \\
\text { al [16] }\end{array}$ & ADNI & KNN & MRI & $\begin{array}{c}\text { Textural } \\
\text { Features } \\
\text { (RROI) }\end{array}$ & NC vs MCI & 63.73 & 55.26 & 67.14 \\
\hline $\begin{array}{c}\text { Casanova et al. } \\
{[17]}\end{array}$ & ADNI & SVM & MRI & cortical & $A D$ vs $\mathrm{NC}$ & 100 & 100 & 100 \\
\hline $\begin{array}{c}\text { Mattsson et al. } \\
{[18]}\end{array}$ & $\begin{array}{c}\text { FINDE } \\
\text { R }\end{array}$ & F-AV & MRI,tPET & $\begin{array}{c}\text { regional } \\
\text { cortical }\end{array}$ & $\mathrm{AD}$ & 93 & & \\
\hline $\begin{array}{c}\text { Das, D et.al. } \\
\text { [19] }\end{array}$ & ADNI & SHIMR & $\begin{array}{c}\text { MRI,CSF,P } \\
\text { ET }\end{array}$ & protein & $A D$ vs NC & 82 & & \\
\hline $\begin{array}{c}\text { Ji, H.,et al. } \\
{[20]}\end{array}$ & ADNI & $\mathrm{CNN}$ & MRI & ROI & AD vs MCI & 100 & 100 & 96 \\
\hline
\end{tabular}

Table 1: Overview of Classification Paradigms for Detection of Alzheimer's disease in 2019 


\begin{tabular}{|c|c|c|c|c|c|c|c|c|}
\hline \multirow{2}{*}{ Author } & \multirow{2}{*}{ Dataset } & \multirow{2}{*}{ Methodology } & \multirow{2}{*}{$\begin{array}{c}\text { Image } \\
\text { modality }\end{array}$} & \multirow{2}{*}{ Features } & \multirow{2}{*}{ Target } & \multicolumn{3}{|c|}{ Performance } \\
\hline & & & & & & Accuracy & Sensitivity & Specificity \\
\hline $\begin{array}{l}\text { Liu et al. } \\
\text { [21] }\end{array}$ & ADNI & $\begin{array}{l}\text { Multi task } \\
\text { multi } \\
\text { channel- } \\
\text { DNN }\end{array}$ & MRI & Patch & $\mathrm{CN}$ vs.AD & 93.7 & 94.6 & 93.2 \\
\hline \multirow{3}{*}{$\begin{array}{l}\text { Sun et al. } \\
\text { [22] }\end{array}$} & \multirow{3}{*}{ ADNI } & \multirow{3}{*}{$\begin{array}{l}\text { Group lasso } \\
\text { SVM }\end{array}$} & \multirow{3}{*}{ sMRI } & \multirow{3}{*}{$\begin{array}{l}\text { VBM } \\
\text { PCC }\end{array}$} & $\mathrm{CN}$ vs. $\mathrm{AD}$ & 95.1 & 93.8 & 83.8 \\
\hline & & & & & CN vs.MCI & 70.8 & 72.1 & 69.1 \\
\hline & & & & & MCI vs.AD & 65.7 & 63.2 & 67.3 \\
\hline $\begin{array}{c}\text { Basaia et al. } \\
{[\underline{23}]}\end{array}$ & ADNI & $\mathrm{CNN}$ & MRI & $\begin{array}{l}\text { GM, } \\
\text { WM, } \\
\text { CSF }\end{array}$ & $\mathrm{CN}$ vs. AD & 98.2 & 98.1 & 98.3 \\
\hline $\begin{array}{l}\text { Lahmiri et al. } \\
\text { [24] }\end{array}$ & ADNI & SVM & MRI & VBM & $\mathrm{CN}$ vs.AD & 100 & 100 & 100 \\
\hline $\begin{array}{c}\text { Lu et al. } \\
{[25]}\end{array}$ & ADNI & MDNN & FDG-PET & ROI & $\mathrm{CN}$ vs. $\mathrm{AD}$ & 93.58 & 91.54 & 95.06 \\
\hline \multirow{2}{*}{$\begin{array}{c}\text { Li et al. } \\
\text { [26] }\end{array}$} & \multirow{2}{*}{ ADNI } & \multirow{2}{*}{ DenseNet } & \multirow{2}{*}{ MRI } & \multirow{2}{*}{ Patch } & $\mathrm{CN}$ vs. AD & 89.5 & 87.9 & 90.8 \\
\hline & & & & & $\mathrm{CN}$ vs MCI & 73.8 & 86.6 & 51.5 \\
\hline \multirow{3}{*}{$\begin{array}{l}\text { Liu et al. } \\
\text { [27] }\end{array}$} & \multirow{3}{*}{ ADNI } & \multirow{3}{*}{$\begin{array}{l}\text { MKBoost } \\
\text { SVM }\end{array}$} & \multirow{3}{*}{ MRI } & \multirow{3}{*}{ Atlas } & $\mathrm{CN}$ vs.AD & 95.37 & 92.49 & 96.08 \\
\hline & & & & & MCI vs.AD & 90.41 & 92.83 & 88.82 \\
\hline & & & & & CN vs.MCI & 86.56 & 90.74 & 84.83 \\
\hline \multirow{3}{*}{$\begin{array}{l}\text { Zheng et al. } \\
\text { [28] }\end{array}$} & \multirow{3}{*}{ ADNI } & \multirow{3}{*}{ MFN } & \multirow{3}{*}{ MRI } & \multirow{3}{*}{ ROI } & $\mathrm{CN}$ vs. AD & 98.7 & 98.59 & 98.79 \\
\hline & & & & & $\begin{array}{l}\text { CN vs. } \\
\text { MCI }\end{array}$ & 97.93 & 98.64 & 96.97 \\
\hline & & & & & $\begin{array}{c}\text { AD vs. } \\
\text { MCI }\end{array}$ & 73.83 & 64.08 & 80.09 \\
\hline \multirow{2}{*}{$\begin{array}{c}\text { Dominguez et al. } \\
{[29]}\end{array}$} & \multirow{2}{*}{$\begin{array}{c}\text { Pitt } \\
\text { Corpus }\end{array}$} & \multirow{2}{*}{$\mathrm{RF}$} & \multirow{2}{*}{ Audio } & $\operatorname{Cov}+\operatorname{lin}$ & $\mathrm{CN}$ vs. $\mathrm{AD}$ & 94 & 100 & 86 \\
\hline & & & & & CN vs.MCI & 87 & 87 & 86 \\
\hline $\begin{array}{l}\text { Cui et al. } \\
{[30]}\end{array}$ & ADNI & ANN+BGRU & MRI & VBM & $\mathrm{CN}$ vs.AD & 89.69 & 86.87 & 92.58 \\
\hline $\begin{array}{c}\text { Jain et al. } \\
\text { [31] }\end{array}$ & ADNI & $\mathrm{CNN}$ & MRI & Entropy & $\mathrm{CN}$ vs. $\mathrm{AD}$ & 99.14 & & \\
\hline $\begin{array}{c}\text { Zhou et al. } \\
\text { [32] }\end{array}$ & ADNI & TrAdaBoost & MRI & Atlas & $\mathrm{CN}$ vs. $\mathrm{AD}$ & 93.75 & 87.5 & 100 \\
\hline & & & & & CN vs.AD & 95.2 & 95.2 & 95.3 \\
\hline $\begin{array}{l}\text { Cheng et al } \\
\text { al. [33] }\end{array}$ & ADNI & rMLTFL & sMRI & VBM & $\mathrm{CN}$ vs.MCI & 82.4 & 86.7 & 73.8 \\
\hline & & & & & MCI vs. AD & 76.7 & 61.4 & 81.8 \\
\hline $\begin{array}{c}\text { Li et al. } \\
{[34]}\end{array}$ & ADNI & $\begin{array}{l}\text { Subspace } \\
\text { alignment }\end{array}$ & fMRI & VBM & $\mathrm{CN}$ vs.AD & 84.6 & 92 & 79 \\
\hline $\begin{array}{c}\text { Kim et al. } \\
{[35]}\end{array}$ & ADNI & MSH-ELM & $\begin{array}{l}\text { MRI,PET, } \\
\text { CSF }\end{array}$ & Atlas & $\mathrm{CN}$ vs. $\mathrm{AD}$ & 97.2 & 98.08 & 94.12 \\
\hline
\end{tabular}

Table 2: Overview of Classification Paradigms for Detection of Alzheimer's disease in 2018 


\begin{tabular}{|c|c|c|c|c|c|c|c|c|}
\hline \multirow{2}{*}{ Author } & \multirow{2}{*}{ Dataset } & \multirow{2}{*}{ Methodology } & \multirow{2}{*}{$\begin{array}{c}\text { Image } \\
\text { modality }\end{array}$} & \multirow{2}{*}{ Features } & \multirow{2}{*}{ Target } & \multicolumn{3}{|c|}{ Performance } \\
\hline & & & & & & Accuracy & Sensitivity & Specificity \\
\hline \multirow{2}{*}{$\begin{array}{l}\text { Liu et al. } \\
{[36]}\end{array}$} & \multirow{2}{*}{ ADNI } & \multirow{2}{*}{$\begin{array}{l}\text { MKBoost, } \\
\text { SVM }\end{array}$} & \multirow{2}{*}{ sMRI } & \multirow{2}{*}{ Atlas } & $\mathrm{CN}$ vs $\mathrm{AD}$ & 94.65 & 95.03 & 91.76 \\
\hline & & & & & MCIvs AD & 89.63 & 91.55 & 86.25 \\
\hline $\begin{array}{c}\text { An, L., et al. } \\
{[37]}\end{array}$ & ADNI & SVM & $\begin{array}{c}\text { MRI + } \\
\text { SNP }\end{array}$ & Discriminative & AD vs. NC & 97.4 & & \\
\hline $\begin{array}{c}\text { Hojjati et al. } \\
{[38]}\end{array}$ & ADNI & SVM & fMRI & PCC , Fscore & $\begin{array}{l}\text { MCIc- } \\
\text { MCInc }\end{array}$ & 91.4 & 83.24 & 90.1 \\
\hline $\begin{array}{c}\text { Lama et al. } \\
{[39]}\end{array}$ & ADNI & PCA, RELM & MRI & Cortical & $\mathrm{CN}$ vs $\mathrm{AD}$ & 77.30 & 62.12 & 79.85 \\
\hline \multirow{3}{*}{$\begin{array}{c}\text { Rupali et al. } \\
{[40]}\end{array}$} & \multirow{3}{*}{ OASIS } & \multirow{3}{*}{$\mathrm{K}-\mathrm{NN}$} & \multirow{3}{*}{ MRI } & \multirow{3}{*}{$\begin{array}{c}\text { Contrast, } \\
\text { Energy, } \\
\text { Homogeneity, }\end{array}$} & AD vs MCI & 92.31 & & \\
\hline & & & & & AD vs NC & 92.75 & & \\
\hline & & & & & MCI vs NC & 83.33 & & \\
\hline $\begin{array}{l}\text { Suk et al. } \\
\text { [41] }\end{array}$ & ADNI & $\begin{array}{c}\text { JLLR } \\
\text { DeepESRNet }\end{array}$ & MRI & ROI & $\mathrm{CN}$ vs $\mathrm{AD}$ & 91.02 & 92.72 & 89.94 \\
\hline $\begin{array}{c}\text { Jha et al. } \\
{[42]}\end{array}$ & OASIS & $\mathrm{PCA}+\mathrm{FFNN}$ & MRI & VBM & $\mathrm{CN}$ vs $\mathrm{AD}$ & 90.06 & 92.0 & 87.76 \\
\hline \multirow{2}{*}{$\begin{array}{c}\text { Lei, et al. } \\
\text { [43] }\end{array}$} & \multirow{2}{*}{ ADNI } & \multirow{2}{*}{ R2DSLR } & & \multirow{2}{*}{ GW,ROI } & AD vs. NC & 97.72 & 97.9 & 91.3 \\
\hline & & & & & MCI vs. NC & 82.6 & 64.3 & 86.6 \\
\hline $\begin{array}{c}\text { Long et al. } \\
{[44]}\end{array}$ & ADNI & SVM & MRI & $\mathrm{MDS}+\mathrm{PCA}$ & $\mathrm{CN}$ vs. AD & 96.5 & 93.85 & 97.78 \\
\hline $\begin{array}{l}\text { Zheng et al. } \\
{[45]}\end{array}$ & ADNI & SVM,RBM & $\begin{array}{l}\text { MRI } \\
\text { PET }\end{array}$ & Atlas & $\mathrm{CN}$ vs $\mathrm{AD}$ & $\begin{array}{l}88.52 \\
\pm 8.61\end{array}$ & $\begin{array}{c}84.60 \\
\pm 15.24\end{array}$ & $\begin{array}{c}92.20 \\
\pm 12.61\end{array}$ \\
\hline \multirow{2}{*}{$\begin{array}{c}\text { Zhang et al. } \\
{[46]}\end{array}$} & \multirow{2}{*}{ ADNI } & \multirow{2}{*}{ SVM } & \multirow{2}{*}{ sMRI } & \multirow{2}{*}{$\begin{array}{l}\text { Landmark- } \\
\text { based Spatial }\end{array}$} & AD vs. $\mathrm{HC}$ & 94.01 & 79.61 & 94.69 \\
\hline & & & & & MCI vs. HC & 85.19 & 90.46 & 59.90 \\
\hline \multirow{2}{*}{$\begin{array}{c}\text { Beheshti et al. } \\
{[47]}\end{array}$} & \multirow{2}{*}{ JADNI } & \multirow{2}{*}{ SVM } & \multirow{2}{*}{ MRI } & VPM & $\mathrm{CN}$ vs $\mathrm{AD}$ & 84.17 & 88.83 & 79.00 \\
\hline & & & & VBM & CN vs MCI & 70.38 & 78.17 & 60.22 \\
\hline $\begin{array}{l}\text { Mathotaarachchi, } \\
\text { et al.[48] }\end{array}$ & ADNI & RUSRF & $\begin{array}{l}\text { MRI, } \\
\text { PET, } \\
\text { CSF }\end{array}$ & Brain voxel & $\begin{array}{c}\text { pMCI vs } \\
\text { AD }\end{array}$ & 84.0 & 70.8 & 86.5 \\
\hline $\begin{array}{c}\text { Asgari et al. } \\
{[49]}\end{array}$ & - & $\mathrm{SVM}+\mathrm{RF}$ & $\begin{array}{l}\text { Word } \\
\text { count }\end{array}$ & LIWC & AD vs.MCI & 74.7 & 67.51 & 72.3 \\
\hline $\begin{array}{c}\text { Lu et al. } \\
{[50]}\end{array}$ & ADNI & RF-RSVM & $\begin{array}{l}\text { FDG- } \\
\text { PET }\end{array}$ & VBM & CN vs.MCI & 90.53 & 90.63 & 93.33 \\
\hline $\begin{array}{c}\text { Cheng, D et al. } \\
{[51]}\end{array}$ & ADNI & $\begin{array}{l}\mathrm{CNN}+ \\
\mathrm{BGRU}\end{array}$ & $\begin{array}{l}\text { FDG- } \\
\text { PET }\end{array}$ & intra-slice & AD vs. NC & 95.28 & 91.00 & 91.40 \\
\hline Cheng & ADNI & MDTFS + & MRI & Atlas & $\mathrm{CN}$ vs $\mathrm{AD}$ & 94.7 & 94.1 & 94.8 \\
\hline et al. [52] & ADNI & MDTC & MRI & Atlas & CN vs MCI & 81.5 & 85.8 & 73.3 \\
\hline $\begin{array}{c}\text { Alam et al. } \\
\text { [53] }\end{array}$ & ADNI & TWSVM & sMRI & DTCWT & $\mathrm{CN}$ vs. AD & 96.88 & 97.72 & 95.61 \\
\hline $\begin{array}{c}\text { Kajal Gulhare } \\
\text { et al [54] }\end{array}$ & OASIS & DNN & MRI & Textural & $\mathrm{AD}$ & 96.61 & & \\
\hline $\begin{array}{c}\text { Arpita et al } \\
{[55]}\end{array}$ & OASIS & ANN & MRI & Textural & AD vs.MCI & $86.8 \%$ & & \\
\hline
\end{tabular}

Table 3: Overview of Classification Paradigms for Detection of Alzheimer's disease in 2017

\section{Critical Evaluation:}

Machine Learning is exploit different of probabilistic and optimization techniques, it plays vital role in field of Medical for diagnosis of disease in early stages for that purpose they use various images imaging modalities of brain. Machine Learning methods are integrated with medical images it will help to Predict Alzheimer's disease in early stage. In above section presented overview of different machine learning classifiers proposed by different researches for detection of Alzheimer's.

These studies are used different classifiers like Support Vector Machine, Deep Learning, Artificial Neural Network, and also use different types of features extraction methods to get the accurate results. Several strategies achieved 
promising prediction accuracies; Even though, being able to identify potential issue Such as pre-processing, selection of image modalities, the number of important attributes for feature selection, experiential Design, validation of model, they are not appropriate on detecting the $\mathrm{AD}$ disease on time for the earlier diagnosis. Through the analysis of studies, the most common problems are extract relevant characteristics and optimal classifier needed for better accuracy with same datasets.

\section{Proposed Model:}

To address these limitations of extract relevant characteristics and optimal classifier, a predictive model is proposed shown in figure1. It Consist of 3 stages 1. Preprocessing 2. Extract relevant characteristics 3. Optimized Classifier. Magnetic resonance imaging (MRI) volumetric measures is a standard tool for the detection of development Alzheimer's Disease (AD) dementia in mild cognitive impairment (MCI). In this study the MRI is data used were acquire from the Alzheimer's Disease Neuroimaging Initiative (ADNI) database.

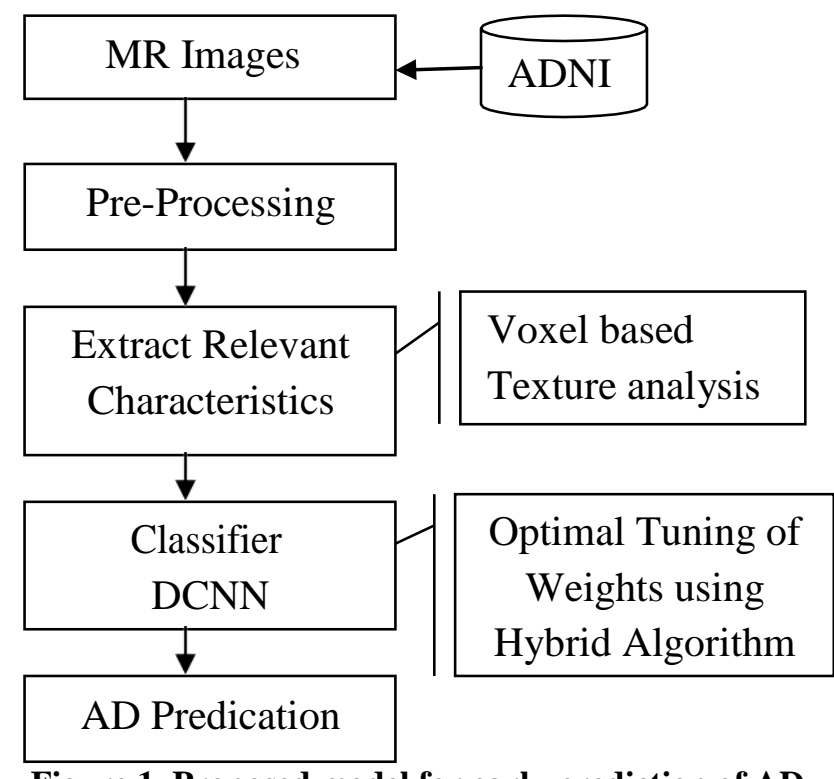

Figure 1. Proposed model for early prediction of AD

The proposed model entails a pre-processing stage for eliminating the issue of class imbalance. It 's important for attributes selection using the machine learning method also help to avoid the problem of too few instances, In Stage 2 perform Voxel based texture analysis of brain MRI, Texture evaluation is a effective quantitative technique for examine Voxel intensities and their interrelationships. Then in stage 3, Optimized Deep Convolution Neural Network (DCNN) will be employed, weight and the activation function in $\mathrm{CNN}$ will be optimally tuned by a new "Hybrid" algorithm, which is the hybridized concept of Grey Wolf Optimizer (GWO) and Dragonfly Algorithm (DA). Finally validate the model by using testing data, model may help better to Prediction of Alzheimer's disease in early stage.

\section{Conclusion:}

This article contributes comparisons and assessment of recent research done in prediction of Alzheimer's disease using machine learning techniques. As Recognized from the review much research has been done in early, but crucial need reaming to identify appropriate features and optimized classifiers need to predict AD in early stages. We proposed predicate model for Alzheimer Disease using MRI, it can extract Voxel based textural features and optimized DCNN classifier prefer. Proposed model may increase accuracy and it help to improve predication of $\mathrm{AD}$ and offset constraints indicate in previous research

\section{References}

[1] Selvakumar, I. Computer aided classification and segmentation of abnormal human brain magnetic resonance images using modified softcomputing techniques.

[2] Kruthika, K. R., Maheshappa, H. D., \& Alzheimer's Disease Neuroimaging Initiative. (2019). Multistage classifier- based approach for Alzheimer's disease prediction and retrieval. Informatics in Medicine Unlocked, 14, 34-42.

[3] What Is Alzheimer's Disease?. National Institute on Aging. (2020). Retrieved 2 July 2020, from http://www.nia.nih.gov/health/what-alzheimers-disease.

[4] 10 Early Signs and Symptoms of Alzheimer's Alzheimer's Disease and Dementia. (2020). Retrieved 2 July 2020, from https://www.alz.org/alzheimersdementia/10_signs.

[5] Clinical Stages of Alzheimer's. Fisher Center for Alzheimer's Research Foundation. (2020). Retrieved 1 July 2020, from https://www.alzinfo.org/understandalzheimers/clinical-stages-of-alzheimers/.

[6] Machine Learning: What it is and why it matters(2018). Sas.com. Retrieved 1 July 2020, from https://www.sas.com/en_us/insights/analytics/machinelearning.html.

[7] Sunil ray, Commonly Used Machine Learning Algorithms(2017)| Data Science. Analytics Vidhya. Retrieved 1 July 2020, from https://www.analyticsvidhya.com/blog/2017/09/commo n-machine-learning-algorithms/.

[8] ML | Semi-Supervised Learning - GeeksforGeeks. GeeksforGeeks. (2020). Retrieved 1 July 2020, from https://www.geeksforgeeks.org/ml-semi-supervisedlearning/

[9] Osiński, B., \& Budek, K. (2018). What is reinforcement learning? The complete guide deepsense.ai.deepsense.ai. Retrieved 3 July 2020, from https://deepsense.ai/what-is-reinforcement-learningthe-complete- guide/.

[10] Jialin Peng, Xiaofeng Zhu, Ye Wang, Le An, and Dinggang Shen. 2019. Structured sparsity regularized 
multiple kernel learning for Alzheimer's disease diagnosis. Pattern Recogn. 88 (2019), 370-382.

[11] Subrata Kar and D. Dutta Majumder. 2019. A novel approach of diffusion tensor visualization-based neuro fuzzy classification system for early detection of Alzheimer's disease. J. Alzheimer's Dis. Rep. 3, 1 (2019),1-18.

[12] Jinhua Sheng, Bocheng Wang, Qiao Zhang, Qingqiang Liu, Yangjie Ma, Weixiang Liu, Meiling Shao, and Bin Chen. 2019. A novel joint HCPMMP method for automatically classifying Alzheimer's and different stage MCI patients. Behav. Brain Res. 365 (2019), 210-221 .

[13] Simeon Spasov, Luca Passamonti, Andrea Duggento, Pietro Lio, Nicola Toschi, Alzheimer's Disease Neuroimaging Initiative, et al. 2019. A parameterefficient deep learning approach to predict conversion from mild cognitive impairment to Alzheimer's disease. NeuroImage 189 (2019), 276-287.

[14] Julian Fritsch, Sebastian Wankerl, and Elmar Nöth. 2019. Automatic diagnosis of Alzheimer's disease using neural network language models. In Proceedings of the IEEE International Conference on Acoustics, Speech and Signal Processing (ICASSP'19). IEEE, 5841-5845.

[15] Gosztolya, G., Vincze, V., Tóth, L., Pákáski, M., Kálmán, J., \& Hoffmann, I. (2019). Identifying mild cognitive impairment and mild Alzheimer's disease based on spontaneous speech using ASR and linguistic features. Computer Speech \& Language, 53, 181-197.

[16] Vaithinathan, K., Parthiban, L., \& Alzheimer's Disease Neuroimaging Initiative. (2019). A novel texture extraction technique with T1weighted MRI for the classification of Alzheimer's disease. Journal of neuroscience methods, 318, 84-99.

[17] Lahmiri, S., \& Shmuel, A. (2019). Performance of machine learning methods applied to structural MRI and ADAS cognitive scores in diagnosing Alzheimer's disease. Biomedical Signal Processing and Control, 52, 414-419.

[18] Mattsson, N., Insel, P. S., Donohue, M., Jögi, J., Ossenkoppele, R., Olsson, T., \& Hansson, O. (2019). Predicting diagnosis and cognition with 18F-AV-1451 tau PET and structural MRI in Alzheimer's disease. Alzheimer's \& Dementia, 15(4), 570-580.

[19] Das, D., Ito, J., Kadowaki, T., \& Tsuda, K. (2019). An interpretable machine learning model for diagnosis of Alzheimer's disease. PeerJ, 7, e6543.

[20] Ji, H., Liu, Z., Yan, W. Q., \& Klette, R. (2019, June). Early diagnosis of Alzheimer's disease using deep learning.In Proceedings of the 2nd International Conference on Control and Computer Vision (pp. $87-$ 91).

[21] Liu, M., Zhang, J., Adeli, E., \& Shen, D. (2018). Joint classification and regression via deep multi-task multichannel learning for Alzheimer's disease diagnosis. IEEE Transactions on Biomedical Engineering, 66(5), 1195-1206.

[22] Zhuo, S., Yuchuan, Q., Boudewijn, P. F., Marius, S., \& Alzheimer's Disease NeuroImaging Initiative. (2018).Integrating spatial- anatomical regularization and structure sparsity into SVM: improving interpretation of Alzheimer's disease classification. Neuroimage, 178, 445-460.

[23] Basaia, S., Agosta, F., Wagner, L., Canu, E., Magnani, G., Santangelo, R., \& Alzheimer's Disease Neuroimaging Initiative. (2019). Automated classification of Alzheimer's disease and mild cognitive impairment using a single MRI and deep neural networks. NeuroImage: Clinical, 21, 101645.

[24] Lahmiri, S., \& Shmuel, A. (2019). Performance of machine learning methods applied to structural MRI and ADAS cognitive scores in diagnosing Alzheimer's disease. Biomedical Signal Processing and Control, 52, 414-419.

[25] Lu, D., Popuri, K., Ding, G. W., Balachandar, R., Beg, M. F., \& Alzheimer's Disease Neuroimaging Initiative.(2018). Multiscale deep neural network based analysis of FDG-PET images for the early diagnosis of Alzheimer's disease. Medical image analysis, 46, 2634.

[26] Li, F., Liu, M., \& Alzheimer's Disease Neuroimaging Initiative. (2018). Alzheimer's disease diagnosis based on multiple cluster dense convolutional networks. Computerized Medical Imaging and Graphics, 70, 101110.

[27] Liu, J., Li, M., Lan, W., Wu, F. X., Pan, Y., \& Wang, J. (2016). Classification of Alzheimer's disease using whole brain hierarchical network. IEEE/ACM transactions on computational biology and bioinformatics, 15(2), 624-632.

[28] Zheng, W., Yao, Z., Xie, Y., Fan, J., \& Hu, B. (2018). Identification of Alzheimer's disease and mild cognitive impairment using networks constructed based on multiple morphological brain features. Biological Psychiatry: Cognitive Neuroscience and Neuroimaging, 3(10), 887-897.

[29] Hernández-Domínguez, L., Ratté, S., Sierra-Martínez, G., \& Roche-Bergua, A. (2018). Computer-based evaluation of Alzheimer's disease and mild cognitive impairment patients during a picture description task Alzheimer's \& Dementia: Diagnosis, Assessment \& Disease Monitoring, 10, 260-268.

[30] Cui, R., Liu, M., \& Li, G. (2018, April). Longitudinal analysis for Alzheimer's disease diagnosis using RNN. In 2018 IEEE 15th International Symposium on Biomedical Imaging (ISBI 2018) (pp. 1398-1401). IEEE.

[31] Jain, R., Jain, N., Aggarwal, A., \& Hemanth, D. J. (2019). Convolutional neural network based Alzheimer's disease classification from magnetic resonance brain images. Cognitive Systems Research, 57, 147-159.

[32] Ke Zhou, Wenguang $\mathrm{He}$, Yonghui $\mathrm{Xu}$, Gangqiang Xiong, and Jie Cai. 2018. Feature selection and transferlearning for Alzheimer's disease clinical diagnosis. Appl. Sci. 8, 8 (2018), 1372.

[33] Cheng, B., Liu, M., Zhang, D., \& Shen, D. (2019). Robust multi-label transfer feature learning for early diagnosis of Alzheimer's disease. Brain imaging and behavior, 13(1), 138-153. 
[34] Wei Li, Yifei Zhao, Xi Chen, Yang Xiao, and Yuanyuan Qin. 2018. Detecting Alzheimer's disease on small dataset: A knowledge transfer perspective. IEEE J. Biomed. Health Info. 23, 3 (2018), 1234-1242.

[35] Kim, J., \& Lee, B. (2018). Identification of Alzheimer's disease and mild cognitive impairment using multimodal sparse hierarchical extreme learning machine. Human brain mapping, 39(9), 3728-3741.

[36] Liu, J., Li, M., Pan, Y., Wu, F. X., Chen, X., \& Wang, J. (2017). Classification of schizophrenia based on individual hierarchical brain networks constructed from structural MRI images. IEEE transactions on nanobioscience, 16(7), 600-608.

[37] An, L., Adeli, E., Liu, M., Zhang, J., Lee, S. W., \& Shen, D. (2017). A hierarchical feature and sample selection framework and its application for Alzheimer's disease diagnosis. Scientific reports, 7(1), 1-11.

[38] Hojjati, S. H., Ebrahimzadeh, A., Khazaee, A., Babajani-Feremi, A., \& Alzheimer's Disease Neuroimaging Initiative. (2017). Predicting conversion from MCI to AD using resting-state fMRI, graph theoretical approach and SVM. Journal of neuroscience methods, 282,69-80.

[39] Lama, R. K., Gwak, J., Park, J. S., \& Lee, S. W. (2017). Diagnosis of Alzheimer's disease based on structural MRI images using a regularized extreme learning machine and PCA features. Journal of healthcare engineering, 2017.

[40] Kamathe, R. S., \& Joshi, K. R. (2018). A Robust Optimized Feature Set Based Automatic Classification Of Alzheimer's Disease From Brain MR Images Using K-NN And Adaboost. ICTACT Journal on Image \& Video Processing, 8(3).

[41] Heung-Il Suk, Seong-Whan Lee, Dinggang Shen, and Alzheimer's Disease Neuroimaging Initiative. 2017. Deep ensemble learning of sparse regression models for brain disease diagnosis. Med. Image Anal. 37 (2017), 101-113.

[42] Jha, D., Kim, J. I., \& Kwon, G. R. (2017). Diagnosis of Alzheimer's disease using dual-tree complex wavelet transform, PCA, and feed- forward neural network. Journal of healthcare engineering, 2017.

[43] Lei, B., Yang, P., Wang, T., Chen, S., \& Ni, D. (2017). Relational-regularized discriminative sparse learning for Alzheimer's disease diagnosis. IEEE transactions on cybernetics, 47(4), 1102-1113.

[44] Xiaojing Long, Lifang Chen, Chunxiang Jiang, Lijuan Zhang, Alzheimer's Disease Neuroimaging Initiative, et.al. 2017. Prediction and classification of Alzheimer's disease based on quantification of MRI deformation. PloS One 12, 3 (2017), e0173372.

[45] Zheng, X., Shi, J., Zhang, Q., Ying, S., \& Li, Y. (2017, April). Improving MRI-based diagnosis of Alzheimer's disease via an ensemble privileged information learning algorithm. In 2017 IEEE 14th International Symposium on Biomedical Imaging (ISBI 2017) (pp. 456-459). IEEE.

[46] Zhang, J., Liu, M., An, L., Gao, Y., \& Shen, D. (2017). Alzheimer's disease diagnosis using landmark-based features from longitudinal structural MR images. IEEE journal of biomedical and health informatics, 21(6), $1607-1616$.

[47] Anbarjafari, G., Beheshti, I., Maikusa, N., Daneshmand, M., Matsuda, H., \& Demirel, H. (2017). Classification of Alzheimer's Disease and Prediction of Mild Cognitive Impairment Conversion Using Histogram-Based Analysis of Patient-Specific Anatomical Brain Connectivity Networks.

[48] Mathotaarachchi, S., Pascoal, T. A., Shin, M., Benedet, A. L., Kang, M. S., Beaudry, T., \& Alzheimer's Disease Neuroimaging Initiative. (2017). Identifying incipient dementia individuals using machine learning and amyloid imaging. Neurobiology of aging, 59,80-90.

[49] Asgari, M., Kaye, J., \& Dodge, H. (2017). Predicting mild cognitive impairment from spontaneous spoken utterances. Alzheimer's \& Dementia: Translational Research \& Clinical Interventions, 3(2), 219-228.

[50] Lu, S., Xia, Y., Cai, W., Fulham, M., Feng, D. D., \& Alzheimer's Disease Neuroimaging Initiative. (2017). Early identification of mild cognitive impairment using incomplete random forest-robust support vector machine and FDG-PET imaging. Computerized Medical Imaging and Graphics, 60, 35-41.

[51] Cheng, D., \& Liu, M. (2017, October). Combining convolutional and recurrent neural networks for Alzheimer's disease diagnosis using PET images. In 2017 IEEE International Conference on Imaging Systems and Techniques(IST) (pp. 1-5). IEEE.

[52] Cheng, B., Liu, M., Shen, D., Li, Z., \& Zhang, D. (2017). Multi-domain transfer learning for early diagnosis of Alzheimer's disease. Neuroinformatics, 15(2), 115-132.

[53] Alam, S., Kwon, G. R., Kim, J. I., \& Park, C. S. (2017). Twin SVM-based classification of Alzheimer's disease using complex dual-tree wavelet principal coefficients and LDA. Journal of healthcare engineering, 2017.

[54] Kajal Gulhare, S.P. Shukla, L. K. Sharma, “Deep Neurak Network Classification Method to Alzheimer's Disease Detection", International Journals of Advanced Research in Computer Science and Software Engineering, Volume-7, Issue-6, pp. 1-4, June 2017. Arpita Raut, Vipul Dalal “ A Survey of Recent Approaches for Detection of Alzheimer's Disease”, International Journal of Engineering Research in Computer Science and Engineering (IJERCSE) Vol 4, Issue 2, February 2017. 